\title{
End-Stage Renal Disease Due to Delayed Diagnosis of Renal Tuberculosis: A Fatal Case Report
}

\author{
Elizabeth De Francesco Daher ${ }^{1,2}$, Geraldo Bezerra da Silva Júnior ${ }^{1}$, Renata Trindade Damasceno², Gustavo Martins dos Santos², \\ Germana Alves Corsino ${ }^{2}$, Sônia Leite da Silva ${ }^{1}$ and Oswaldo Augusto Gutiérrez-Adrianzén ${ }^{1}$ \\ ${ }^{1}$ Department of Internal Medicine, Medicine School, Federal University of Ceará; ${ }^{2}$ Division of Nephrology, General Hospital of Fortaleza, \\ Secretary of Public Health of Ceará; Fortaleza, CE, Brazil
}

\begin{abstract}
Renal TB is difficult to diagnose, because many patients present themselves with lower urinary symptoms which are typical of bacterial cystitis. We report a case of a young woman with renal TB and ESRD. She was admitted with complaints of adynamia, anorexia, fever, weight loss, dysuria and generalized edema for 10 months. At physical examination she was febrile $\left(39^{\circ} \mathrm{C}\right)$, and her abdomen had increased volume and was painful at palpation. Laboratorial tests showed serum urea $=220 \mathrm{mg} / \mathrm{dL}$, creatinine $=6.6 \mathrm{mg} / \mathrm{dL}$, hemoglobin $=7.9 \mathrm{~g} / \mathrm{dL}$, hematocrit $=24.3 \%$, leukocytes $=33,600 / \mathrm{mm}^{3}$ and platelets $=664,000 / \mathrm{mm}^{3}$. Urinalysis showed an acid urine $(\mathrm{pH}=5.0)$, leukocyturia $(2+/ 4+)$ and mild proteinuria $(1+/ 4+)$. She was also oliguric (urinary volume $<400 \mathrm{~mL} /$ day). Abdominal echography showed thick and contracted bladder walls and heterogeneous liquid collection in the left pelvic region. Two laparotomies were performed, in which abscess in pelvic region was found. Anti-peritoneal tuberculosis treatment with rifampin, isoniazid and pyrazinamide was started. During the follow-up, the urine culture was found to be positive for $M$. tuberculosis. Six months later the patient had complaints of abdominal pain and dysuria. New laboratorial tests showed serum urea $=187 \mathrm{mg} / \mathrm{dL}$, creatinine $=8.0 \mathrm{mg} / \mathrm{dL}$, potassium $=6.5 \mathrm{mEq} / \mathrm{L}$. Hemodialysis was then started. The CT scan showed signs of chronic nephropathy, dilated calyces and thinning of renal cortex in both kidneys and severe dilation of ureter. The patient developed neurologic symptoms, suggesting tuberculous meningoencephalitis, and died despite of support measures adopted. The patient had ESRD due to secondary uropathy to prolonged tuberculosis of urinary tract that was caused by delayed clinical and laboratorial diagnosis, and probably also due to inadequate antituberculous drugs administration.
\end{abstract}

Key-Words: Tuberculosis, renal tuberculosis, extrapulmonary tuberculosis, end-stage renal disease, chronic renal failure.

Tuberculosis (TB) is a current problem of public health, with an estimated global incidence of 8 to 10 million/year [1]. Since the advent of HIV-pandemia TB presents an increasing incidence and still remains the most common worldwide cause of mortality of infectious disease [2-4].

According to recent studies, genitourinary TB is the second most common form of extrapulmonary disease after peripheral lymphadenopathy $[1,3,5]$. Other sites of extrapulmonary infection include pleura, pericardium, and osteoarticular areas, but any organ can be involved [3].

Renal TB is difficult to diagnose, because many patients present themselves with lower urinary symptoms which are typical of conventional bacterial cystitis [1,6]. The suspicions of TB arise when there is no response to antibiotics or when urinalysis shows the presence of leukocyturia in the absence of bacteriuria $[1,6,7]$.

The occurrence of extrapulmonary TB is not common in HIV-negative patients, and the development of end-stage renal disease (ESRD) caused by TB is even less common, what makes this report rare in medical literature [1]. After the patient's family consent, we report a fatal case of a young woman with renal TB and ESRD.

Received on 16 September 2006; revised 27 November 2006.

Address for correspondence: Dr. Elizabeth De Francesco Daher Rua Vicente Linhares 1198. Zip code: 60270-135. Fortaleza, CE, Brazil. Phone / Fax: 558532249725 / 558532613777 E-mail: geraldobezerrajr@yahoo.com.br, ef.daher@uol.com.br.

The Brazilian Journal of Infectious Diseases 2007;11(1):169-171. (C) 2007 by The Brazilian Journal of Infectious Diseases and Contexto Publishing. All rights reserved.

\section{Case Report}

A 32-year-old female, previously healthy, was admitted to our hospital with complaints of adynamia, anorexia, fever, weight loss, dysuria and generalized edema for 10 months. At physical examination she was icteric $(2+/ 4+)$, pale $(3+/ 4+)$, febrile $\left(39^{\circ} \mathrm{C}\right)$, tachypneic, with cardiac auscultation showing pancardiac systolic murmur $(1+/ 4+)$, abdomen with increased volume and painful at palpation. The laboratorial analysis evidenced serum urea $=220 \mathrm{mg} / \mathrm{dL}$, creatinine $=6.6 \mathrm{mg} / \mathrm{dL}$, hemoglobin $=7.9 \mathrm{~g} / \mathrm{dL}$, hematocrit $=24.3 \%$, leukocytes $=33,600 /$ $\mathrm{mm}^{3}$ and platelets $=664,000 / \mathrm{mm}^{3}$. Urinalysis showed an acid urine $(\mathrm{pH}=5.0)$, leukocyturia $(2+/ 4+)$ and mild proteinuria $(1+/$ $4+$ ). She was also oliguric (urinary volume $<400 \mathrm{~mL} /$ day). Abdominal echography showed thick and contracted bladder walls and heterogeneous liquid collection in the left pelvic region (around 800-900 mL).

The suspicion of pelvic abscess was then raised, and treatment with cefepime, ciprofloxacin and metronidazole was started. A urine culture was requested. Seventeen days after admission laparotomy was performed, in which citrine ascitic liquid (around 1,000mL) and gelatinous collection in left pelvic region were found. The bladder walls were thick, contracted and adherent to abdominal wall, suggesting an inflammatory process. The biochemical examination of ascitic liquid showed proteins $=1.5 \mathrm{~g} / \mathrm{dL}$, albumin $=0.8 \mathrm{~g} / \mathrm{dL}$, negative bacterioscopy and negative acid-fast bacilli staining. Anti-HBc, HBsAg, antiHCV, VDRL, anti-HIV, antinuclear antibodies and lupus anticoagulant were all negative. Serum complement was also normal. Two weeks after the surgery the patient was complaining of diffuse abdominal pain and presented a 
palpable abdominal mass in the left iliac region. The ultrasound showed a cystic mass, with a mean volume of $987 \mathrm{~mL}$. Another laparotomy was performed, in which many bowel adherences and caseous-like lesions in bowel walls and in visceral peritoneum were found. The histopathological examination led to the diagnosis of renal, ureteral, vesical and peritoneal tuberculosis. Anti-peritoneal tuberculosis treatment with rifampin, isoniazid and pyrazinamide was started.

The patient became clinical stable and was discharged, with a therapeutic scheme of rifampin - $600 \mathrm{mg} /$ day, isoniazid - $200 \mathrm{mg}$ /day and pyrazinamide - $2 \mathrm{~g} /$ day for two months, and rifampin plus isoniazid for four months. The laboratorial tests in this occasion showed serum urea $=63 \mathrm{mg} / \mathrm{dL}$, creatinine $=1.6$, hemoglobin $=10.9 \mathrm{~g} / \mathrm{dL}$, hematocrit $=33.5 \%$, leukocytes $=24,000 /$ $\mathrm{mm}^{3}$, platelets $=541,000 / \mathrm{mm}^{3}, A S T=24 \mathrm{IU} / \mathrm{L}$, total bilirrubin $=0.76 \mathrm{~g} / \mathrm{dL}$. During the follow-up the urine culture was found to be positive for Mycobacterium tuberculosis.

Six months later the patient had complaints of abdominal pain and dysuria. New laboratorial tests showed serum urea $=187 \mathrm{mg} / \mathrm{dL}$, creatinine $=8.0 \mathrm{mg} / \mathrm{dL}$, potassium $=6.5 \mathrm{mEq} / \mathrm{L}$. It was started hemodialysis, three times a week. The ultrasound showed a septed cystic mass filling all the left side of the abdomen and bilateral hydronephrosis. The CT scan showed signs of chronic nephropathy, dilated calyces and thinning of renal cortex in both kidneys (Figure 1) and severe dilation of ureter-megaureter (Figure 2). The patient developed neurologic symptoms (convulsions, vomiting and disorientation), suggesting tuberculous meningoencephalitis. She was transferred to the intensive care unit (ICU), but died despite of support measures adopted.

\section{Discussion}

We reported a rare case of end-stage renal disease caused by renal tuberculosis. It is believed that $8 \%$ to $15 \%$ of patients

Figure 1. Axial non-contrasted image of CT scan showing enlargement of both kidneys, cortical thinning with lowdensity, markedly dilated calyces (arrows).

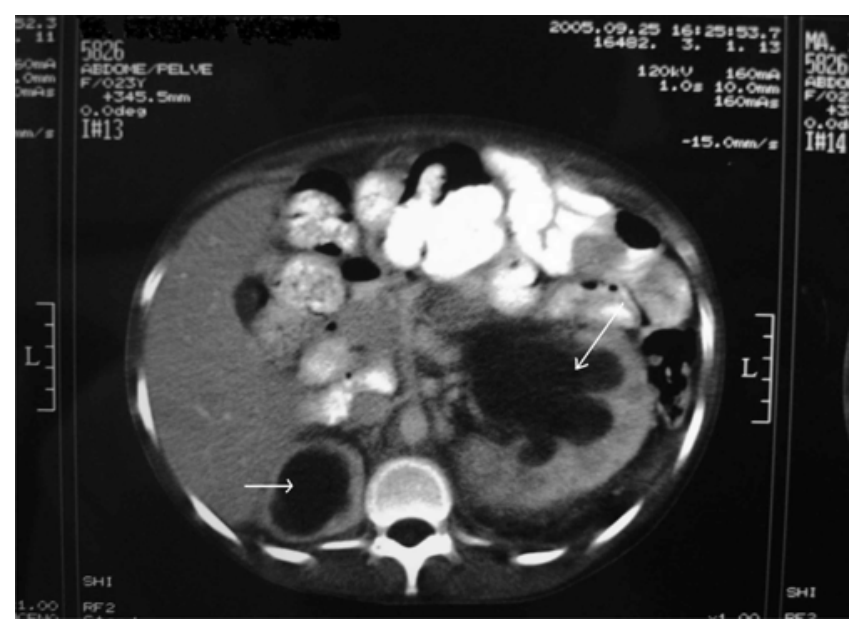

with pulmonary tuberculosis develop infection in the genitourinary system [5]. TB can cause direct renal damage, or secondary renal amyloidosis [7]. Renal tuberculosis, the second most frequent kind of extrapulmonary tuberculosis [1], is a common missed genitourinary infection that should be considered in any case of urinary symptoms, like pyuria or hematuria, which does not respond to common antibacterial drugs [8]. This condition is always secondary to a pulmonary infection, though in the majority of cases this primary focus has healed or is quiescent. The bacilli can reach other organs, including the kidneys, via the hematogenous route $[2,8]$.

In this case the patient had a chronic history of unspecific symptoms, like adynamia, anorexia, fever, weight loss and dysuria, but TB was not initially suspected. The onset of clinically evident genitourinary tuberculosis is often insidious, with dysuria and gross hematuria being the most common symptoms [6]. The time between infection and the appearance of symptoms can be long, varying from 5 to 40 years [2,6]. Some patients are asymptomatic, and pyuria and/or microscopic hematuria are found incidentally. These two abnormalities are present in more than $90 \%$ of cases [7]. Our patient presented no evidence of pulmonary infection, but as we live in an endemic area of TB, for any patient presenting with fever of unknown origin the diagnostic hypothesis of renal TB must always be raised.

The laboratorial diagnosis of renal tuberculosis is difficult, because it is hard to identify the organisms in the urine and there is a variety of clinical and radiological presentations [4,7]. Bennani et al. [9], studying 109 cases of renal tuberculosis, have found bacilli in the urine of 41 patients (38\%). This is comprehensible because bacilli are shed into the urine intermittently, resulting in only $30 \%$ to $40 \%$ of single urine specimens to be positive in patients with active disease [7]. In the investigation of renal TB, at least three first-morning-

Figure 2. Sequential axial non-contrasted image of CT scan showing a severe dilation of ureter, megaureter (arrows).

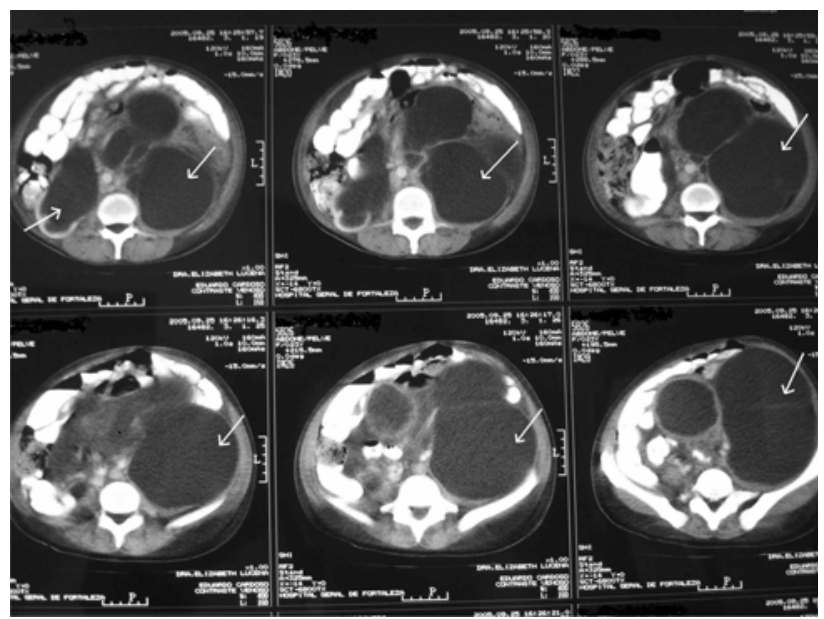


void urine samples should be collected for acid-fast bacilli staining and mycobacterial cultures [2]. The urine is classically sterile by routine culture, with concurrent bacteriuria occurring in some cases. The urinary $\mathrm{pH}$ is usually acid, which is an important feature to differentiate from the common urinary infections [6]. In this case the urinalysis performed at admission found acid urine, with leukocyturia and proteinuria. The findings of dysuria, sterile pyuria, hematuria and characteristically radiological features are highly suggestive of renal tuberculosis, but it has to be confirmed through urine culture, which is the gold standard method for establishing the diagnosis [7]. However, the finding of acid-fast bacilli in the urine with a Ziehl-Neelsen stain or fluorescent dye techniques is not pathognomonic for this disorder, since nonpathogenic mycobacteria may occasionally be present [7]. In the case presented here, $M$. tuberculosis has not been found through acid-fast bacilli staining. The diagnosis was confirmed only after ten sequential urine cultures. In the present case the urine culture was positive for $M$. tuberculosis.

Intravenous urography can also be used in the investigation of renal tuberculosis, which can show characteristically signs, mainly in the ureter or the pielocalycial region $[8,9]$. Erosion of the tips of the calyces, blunting of the calyces or overt papillary necrosis, and parenchymal scarring and calcification can be seen. In the present case, the computed tomography showed important abnormalities, such as calyectasias, thinning of renal cortex and severe dilation of ureter, which is strongly suggestive of TB. Differential diagnosis for the imaging appearance of renal TB includes chronic pyelonephritis, papillary necrosis, medullary sponge kidney, calyceal diverticulum, renal cell carcinoma, transitional cell carcinoma, and xantogranulomatous pyelonephritis [2].

Our patient presented renal failure and ESRD caused by renal TB, which is not frequent $[10,11]$. There are two mechanisms through which TB can cause renal failure intrinsic infection within renal parenchyma or obstructive uropathy [5]. Intrinsic infection is characterized by progressive inflammatory changes in renal parenchyma, which can progress and develop caseous necrosis and fibrosis of the collecting system and ureter. These changes induce a clinical picture of obstructive uropathy [5]. The end-result of diffuse disease in the kidney is destruction, loss of function, and calcification of the entire organ [4].
The treatment of renal tuberculosis consists of antituberculous drugs. The first line drugs include rifampin, isoniazid and pyrazinamide, ethambutol and streptomycin [5]. Antituberculous agents are successful in eradicating active infection in almost all patients. Ureteral strictures may progress during treatment due to scarring and subsequent narrowing of the lumen. It is recommended to perform intravenous pyelogram or renal ultrasound every six months for the first two years in patients with ureteral strictures to detect possible urinary tract obstruction [7]. The treatment of renal TB should be continued for at least six months [5]. In the present case the patient was treated for six months, with partial recovery, but presented a relapse. We could attribute this relapse to a possible patient's non-adherence to treatment, resistance of $M$. tuberculisis to the drugs or the degree of infection, which showed to be severe, as evidenced in CT. In conclusion, the patient had ESRD due to secondary uropathy to prolonged tuberculosis of urinary tract that was caused by delayed clinical and laboratorial diagnosis, and probably also due to inadequate antituberculous drugs administration.

\section{References}

1. Eastwood J.B., Corbishley C.M., Grange J.M. Tuberculosis and the kidney. J Am Soc Nephrol 2001;12:1307-14.

2. Gibson M.S., Puckett M.L., Shelly M.E. Renal tuberculosis. Radiographics 2004;24:251-6.

3. Golden M.P., Vikram H.R. Extrapulmonary tuberculosis: an overview. Am Fam Physician 2005;72:1761-8.

4. Muttarak M., Chiangmai W.N., Lojanapiwat B. Tuberculosis of the genitourinary tract: imaging features with pathological correlation. Singapore Med J 2005;46:568-75.

5. Wise G.J., Merella V.K. Genitourinary manifestations of tuberculosis. Urol Clin North Am 2003;30:111-21.

6. Afiune J.B. Tuberculose extrapulmonar. In: Veronesi R., Focaccia R. (eds). Tratado de Infectologia. 2. ed. São Paulo: Atheneu, 2002, p. 964-9.

7. Rose B.D. Renal disease in tuberculosis. UpToDate, 2002. In: http://www.uptodate.com/.

8. Williams R.D., Cooper C.S., Donovan J.F., Jr. Urology. In: Way LW, Doherty GM (eds). Current surgical diagnosis and treatment. 11. ed. New York: McGraw-Hill, 2003, p.1013-84.

9. Bennani S., Fekak H., Hafiani A., et al. La tuberculose urogénitale. À propos de 109 cas. Méd Mal Infect 1999;29:19-25.

10. Conte G., Iavanore M., Santorelli V., DeNicola L. Acute renal failure of unknown origin. Don't forget renal tuberculosis. Nephrol Dial Transplant 1997;12:1260-1.

11. Khoury N., Minot G., Vigneau C., et al. An unusual presentation of renal failure. Nephrol Dial Transplant 2002;17:679-81. 